\title{
GRADED PRIMAL SUBMODULES OF GRADED MODULES
}

\author{
Ahmad Yousefian Darani
}

\begin{abstract}
Let $G$ be an abelian monoid with identity $e$. Let $R$ be a $G$-graded commutative ring, and $M$ a graded $R$-module. In this paper we first introduce the concept of graded primal submodules of $M$ and give some basic results concerning this class of submodules. Then we characterize the graded primal ideals of the idealization $R(+) M$.
\end{abstract}

A grading on a ring and its modules usually aids computations by allowing one to focus on the homogeneous elements, which are presumably simpler or more controllable than random elements. However, for this to work one needs to know that the constructions being studied are graded. One approach to this issue is to redefine the constructions entirely in terms of the category of graded modules and thus avoid any consideration of non-graded modules or non-homogeneous elements; Sharp gives such a treatment of attached primes in [12]. Unfortunately, while such an approach helps to understand the graded modules themselves, it will only help to understand the original construction if the graded version of the concept happens to coincide with the original one. Therefore, notably, the study of graded modules is very important.

Throughout this paper all rings are considered to be commutative with identity. The concept of primal ideals has been introduced by Fuchs [8]. Later Dauns [2] generalized the concept of primal ideals to modules. Let $R$ be a commutative ring, $M$ an $R$-module and $N$ a submodule of $M$. An element $r \in R$ is called prime to $N$ if $r m \in N(m \in M)$ implies that $m \in N$. $N$ is said to be primal if the set $S(N)$ of elements of $R$ that are not prime to $N$ forms an ideal; this ideal is always a prime ideal of $R$, called the adjoint ideal $P$ of $N$. In this case we also say that $N$ is a $P$-primal submodule. In this paper we define the concept of graded primal submodule of a graded module $M$ over the $G$-graded ring $R$ and then we study this class of submodules.

We first introduce the notations and definitions that we will use throughout. Let $G$ be an arbitrary commutative monoid with identity $e$. By a $G$-graded

Received January 24, 2010; Revised January 30, 2011.

2010 Mathematics Subject Classification. 13A02, 16W50.

Key words and phrases. graded primary submodule, graded prime submodule, graded primal submodule. 
commutative ring we mean a commutative $\operatorname{ring} R$ with a non-zero identity together with a direct sum decomposition (as an additive group) $R=\oplus_{g \in G} R_{g}$ with the property that $R_{g} R_{h} \subseteq R_{g h}$ for all $g, h \in G$. We also denote this by $(R, G)$. Also we write $h(R)=\cup_{g \in G} R_{g}$. The summands $R_{g}$ are called homogeneous components and elements of these summands are called homogeneous elements of $R$. If $a \in R$, then $a$ can be written uniquely as $a=\sum_{g \in G} a_{g}$ where $a_{g}$ is the component of $a$ in $R_{g}$. In this case $R_{e}$ is a sub-ring of $R$ and $1_{R} \in R_{e}$. Let $I$ be an ideal of $R$. Then $I$ is a graded ideal of $(R, G)$ if $I=\oplus_{g \in G}\left(I \cap R_{g}\right)$. Let $R=\oplus_{g \in G} R_{g}$ and $S=\oplus_{g \in G} S_{g}$ be $G$-graded rings and let $h \in G$. A ring homomorphism $\varphi: R \rightarrow S$ is called homogeneous of degree $h$ if $\varphi\left(R_{g}\right) \subseteq S_{g h}$ for every $g \in G$. In the following $R=\oplus_{g \in G} R_{g}$ will a $G$-graded commutative ring.

Let $I$ be a graded ideal of $(R, G)$. $I$ is called a graded prime ideal if $I \neq R$ and whenever $r s \in I$, we have $r \in I$ or $s \in I$, where $r, s \in h(R)$. I is called a graded maximal ideal of $(R, G)$ if $I \neq R$ and there is no graded ideal $J$ of $(R, G)$ with $I \subset J \subset R$. The graded radical of $I$ is denoted by $\operatorname{Gr}(I)$ and consists of all $x \in R$ such that, for each $g \in G$, there exists a positive integer $n_{g}$ with $x_{g}^{n_{g}} \in I$. Note that if $r$ is a homogeneous element of $(R, G)$, then $r \in G r(I)$ if and only if $r^{n} \in I$ for some positive integer $n$ (c.f. $[3,11]$ ).

A $G$-graded $R$-module (or simply graded module) is an $R$-module $M$ such that $M=\oplus_{g \in G} M_{g}$ where every $M_{g}$ is an additive subgroup of $M$, and for every $g, h \in G$ we have $R_{g} M_{h} \subseteq M_{g h}$. Since $R_{e} M_{h} \subseteq M_{h}$ we see that every $M_{h}$ is an $R_{e}$-submodule of $M$. The elements of $h(M)=\cup_{g \in G} M_{g}$ are called the homogeneous elements of $M$. A nonzero element $m \in M_{g}$ is said to be homogeneous of degree $g$, and we write $\operatorname{deg}(m)=g$. Every $m \in M$ can be uniquely represented as a sum $m=\sum_{g \in G} m_{g}$ with $m_{g} \in M_{g}$ and finitely many nonzero $m_{g}$. The nonzero elements $m_{g}$ in this sum are called the homogeneous components of $m$. An $R$-submodule $N$ of $M$ is said to be a graded submodule if for every $n \in N$ all its homogeneous components are also in $N$, i.e., $N=$ $\sum_{g \in G}\left(N \cap M_{g}\right)$. For a graded submodule $N$ of $M$ we may define a graded factor structure on $M / N$ be defining a grading as follows: $(M / N)_{g}=\left(M_{g}+N\right) / N$ for $g \in G$. The reader is referred to [10] for undefined terms and notation.

In Section 1, we give some preliminary results on graded primal submodules. Every graded prime and every graded primary submodule is graded primal. The graded $R$-module $M$ is called graded $P$-module provided that every proper graded submodule of $M$ can be written as a finite product of graded primal ideals of $R$ and a graded primal submodule of $M$. The graded ring $R$ is called graded $P$-ring if it is graded $P$-module as a graded $R$-module. In Theorem 1.9 we prove that if $M$ is a faithful graded finitely generated multiplication $R$ module and if $R$ is a graded $P$-ring, then $M$ is a graded $P$-module. Let $G$ be an abelian group and let $R$ be a $G$-graded commutative ring, and $M$ a graded $R$ module. In Section 2 we discuss on the relationships between the graded primal submodules of $M$ and the graded primal submodules of the graded $R_{S}$-module 
$M_{S}$ where $S$ is a multiplicatively closed subset of homogeneous elements of $R$. It is shown in Theorem 2.5 that there is a one-to-one correspondence between the graded $P$-primal submodules of $M$ and the graded $P_{S}$-primal submodules of $M_{S}$ in which $P$ a graded prime ideal of $R$ and $S$ a multiplicatively closed subset of homogeneous elements of $R$ with $P \cap S=\emptyset$. In Section 3 we consider the Nagata's principle of idealization $R(+) M$ of the graded $R$-module $M$. Then we characterize graded primal submodules of $R(+) M$.

\section{Graded primal submodules}

We start with the following definition:

Definition. Let $R$ be a $G$-graded ring, $M$ a graded $R$-module and $N$ a graded submodule of $M$. Then

(1) An element $a \in h(R)$ is called homogeneous prime to $N$ if $a m \in N$, with $m \in h(M)$, implies that $m \in N$.

(2) An element $a=\sum_{g \in G} a_{g} \in R$ is called $G$-prime to $N$ if at least one homogeneous component $a_{g}$ of $a$ is homogeneous prime to $N$.

Remark 1.1. Let $R$ be a $G$-graded ring and $M$ a graded $R$-module. Let $N$ be a graded submodule of $M$. By definition, an element $a=\sum_{g \in G} a_{g} \in R$ is not $G$-prime to $N$ if, for every $g \in G$, there exists $m_{g^{\prime}} \in h(M) \backslash N$ with $a_{g} m_{g^{\prime}} \in N$. Let $G=\mathbb{Z}_{2}, R_{0}=\mathbb{Z}, R_{1}=i \mathbb{Z}$. Then $R=\mathbb{Z}[i]=R_{0} \oplus R_{1}$ is a graded ring. Consider $R$ as a graded $R$-module. Then the submodule $N=6 R$ is a graded submodule of $R$. As $2,3 \in h(R) \backslash N$ with $2.3=6 \in I$ and 2.(3i) $=6 i \in I$, 2 and $3 i$ are not homogeneous prime to $N$. Thus $2+3 i$ is not $G$-prime to $N$. Furthermore, if there is $x=a+i b \in R$ with $(2+3 i)(a+i b) \in N$, then $2 a-3 b$ and $2 b+3 a$ both belong to $6 \mathbb{Z}$. This implies that $a, b \in 6 \mathbb{Z}$, that is, $x=a+i b \in N$. Therefore, $a$ is prime to $N$ while it is not $G$-prime to $N$. This example shows that the concepts "prime to $N$ " and " $G$-prime to $N$ " are different.

Remark 1.2. Let $R$ be a $G$-graded commutative ring and $N$ a graded submodule of a graded $R$-module $M$. Denote by $g(N)$ the set of all homogeneous elements of $R$ that are not homogeneous prime to $N$ and by $G(N)$ the set of all elements of $R$ that are not $G$-prime to $N$. Then:

(1) $g(N) \subseteq G(N)$, and each element of $G(N)$ is a sum of elements in $g(N)$.

(2) $G(N)$ need not be a graded ideal of $R$.

(3) If $a \in h(R)$ is not homogeneous prime to $N$, then $a$ is not prime to $N$; hence $g(N) \subseteq S(N)$.

Lemma 1.3. Let $R$ be a $G$-graded ring and $M$ a graded $R$-module. If $N$ is a proper graded submodule of $M$, then

(1) $\left(N:_{R} M\right) \subseteq G(N)$.

(2) $G\left(\left(N:_{R} M\right)\right) \subseteq G(N)$. 
Proof. (1) Pick an element $a \in h(R)$ such that $a \in\left(N:_{R} M\right)$. Since $N$ is a proper submodule of $M,\left(N:_{R} M\right)$ is a proper ideal of $R$. So $1_{R} \in h(R) \backslash\left(N:_{R}\right.$ $M)$. Now $a=a .1_{R} \in\left(N:_{R} M\right)$ implies that $a$ is not homogeneous prime to $N$. Hence, by Remark 1.2, $a \in g(N) \subseteq G(N)$; and hence $\left(N:_{R} M\right) \subseteq G(N)$.

(2) Let $a \in h(R) \cap G\left(\left(N:_{R} M\right)\right)$. Then, there exists $b \in h(R) \backslash\left(N:_{R} M\right)$ with $a b \in\left(N:_{R} M\right)$. As $b \notin\left(N:_{R} M\right)$, there exists $m \in h(M)$ with $b m \notin N$. Now it follows from $a b m \in N$ with $b m \in h(M)-N$ that $a$ is not homogeneous prime to $N$. Hence $a \in g(N) \subseteq G(N)$ by Remark 1.2. This implies that $G\left(\left(N:_{R} M\right)\right) \subseteq G(N)$.

Theorem 1.4. Let $R$ be a $G$-graded commutative ring, $M$ a graded $R$-module and $N$ a graded submodule of $M$. If $G(N)$ is an ideal of $R$, then it is a graded prime ideal of $R$.

Proof. Suppose that $a=\sum_{g \in G} a_{g} \in G(N)$. Then, by Remark 1.2, $a_{g} \in g(N) \subseteq$ $G(N)$ for every $g \in G$. Therefore $G(N)$ is a graded ideal of $R$. Now suppose that $a b \in G(N)$ for some $a, b \in h(R)$ but $a \notin G(N)$. So $a b$ is not homogeneous prime to $N$. Hence $(a b) m \in N$ for some $m \in h(M)-N$. As $a \notin G(N), a$ is homogeneous prime to $N$. Thus from $a(b m) \in N$ we get $b m \in N$. This implies that $b$ is not homogeneous prime to $N$, that is, $b \in g(N) \subseteq G(N)$. Consequently $G(N)$ is a $G$-prime ideal of $R$.

Definition. (1) Let $R$ be a $G$-graded commutative ring, $M$ a graded $R$-module and $N$ a graded submodule of $M . N$ is called a graded primal submodule of $M$ if $N \neq M$ and the set $P=G(N)$ of all elements of $R$ that are not $G$-prime to $N$ forms an ideal of $R$. By Theorem 1.4, this ideal is always a graded prime ideal, called the adjoint graded prime ideal of $N$. In this case we also say that $N$ is a graded $P$-primal submodule of $M$.

(2) $M$ is called graded primal if its zero submodule is graded primal. The graded ring $R$ is called graded primal if it is graded primal as an $R$-module.

Definition. Let $R$ be a $G$-graded commutative ring and $M$ a graded $R$-module. An element $a=\sum_{g \in G} a_{g} \in R$ is said to be a graded zero-divisor on $M$ if, for every $g \in G$, there exists a non-zero element $m_{g^{\prime}} \in h(M)$ with $a_{g} m_{g^{\prime}}=0$. We denote by $G z(M)$ the set of all graded zero-divisors of $R$ on $M$.

Remark 1.5. Let $R$ be a $G$-graded commutative ring, $P$ an ideal of $R, M$ a graded $R$-module and $N$ a graded submodule of $M$. On can easily check that $G(N)=G z(M / N)$, where $M / N$ is considered as a graded $R /\left(N:_{R} M\right)$ module. Therefore, $N$ is a graded $P$-primal submodule of $M$ if and only if $\left(N:_{R} M\right) \subseteq P$ and $G z(M / N)=P /\left(N:_{R} M\right)$.

Let $R$ be a $G$-graded ring, $M$ a graded $R$-module and let $N$ be a graded submodule of $M$. We say that $N$ is a graded prime submodule of $M$ if $N \neq M$; and whenever $a \in h(R)$ and $m \in h(M)$ with $a m \in N$, then either $m \in N$ or $a \in\left(N:_{R} M\right) . N$ is called a graded primary submodule of $M$ if $N \neq M$; and whenever $a \in h(R)$ and $m \in h(M)$ with $a m \in N$, then either $m \in N$ or 
$a^{k} \in\left(N:_{R} M\right)$ for some positive integer $k$. If $N$ is a graded primary submodule of $M$, then $P=\operatorname{Gr}\left(\left(N:_{R} M\right)\right)$ is a graded prime ideal of $R$. In this case we say that $N$ is graded $P$-primary (see [4]).

Theorem 1.6. Let $R$ be a G-graded commutative ring and $M$ a graded $R$ module. Then

(1) Every graded primary submodule of $M$ is graded primal.

(2) Every graded prime submodule of $M$ is graded primal.

Proof. (1) Let $Q$ be a graded $P$-primary submodule of $M$. We claim that $P=G(Q)$. Assume that $a \in G(Q)$. Then, for every homogeneous component $a_{g}$ of $a$, there exists $m \in h(M) \backslash Q$ with $a_{g} \in Q$. As $Q$ is graded $P$-primary, we have $a_{g} \in \operatorname{Gr}\left(\left(Q:_{R} M\right)\right)=P$, so $a \in P$. Hence $G(Q) \subseteq P$. Conversely, assume that $b=\sum_{g \in G} b_{g} \in P=\operatorname{Gr}\left(\left(Q:_{R} M\right)\right)$. If $b \in\left(Q:_{R} M\right)$, then $b \in G(Q)$ by Lemma 1.3. So assume that $b \in G r(Q) \backslash\left(Q:_{R} M\right)$. Let $g \in G$. If $b_{g} \in\left(Q:_{R} M\right)$, then $b_{g} \in g(Q)$. If $b_{g} \notin\left(Q:_{R} M\right)$, there exists a smallest positive integer $n>1$ for which $b_{g}^{n} \in\left(Q:_{R} M\right)$. In this case $b_{g} b_{g}^{n-1} \in\left(Q:_{R} M\right)$ with $b_{g}^{n-1} \in h(R) \backslash\left(Q:_{R} M\right)$ implies that $b_{g} \in g\left(\left(Q:_{R} M\right)\right)$. Therefore $b \in$ $G\left(\left(Q:_{R} M\right)\right) \subseteq G(Q)$ by Lemma 1.3 , that is, $P \subseteq G(Q)$. Therefore $G(Q)=P$, and hence $Q$ is graded $P$-primal.

(2) It follows from (1) since every graded prime submodule is graded primary.

Let $R$ be a $G$-graded commutative ring. A graded $R$-module $M$ is called a graded multiplication module provided that for each graded submodule $N$ of $M$, $N=I M$ for some graded ideal $I$ of $R[6]$. It is easy to show that if $N$ is a graded submodule of a graded multiplication module $M$, then $N=\left(N:_{R} M\right) M$. A graded $R$-module $M$ is called graded finitely generated if $M=\sum_{i=1}^{n} R x_{g_{i}}$ where $x_{g_{i}} \in h(M)(1 \leq i \leq n)$.

Remark 1.7 ([5, Lemma 3.10] and [13, Theorem 10]). If $M$ is a graded finitely generated multiplication module over a $G$-graded $\operatorname{ring} R$ and $I$ is a graded ideal of $R$ containing $\left(0:_{R} M\right)$, then $\left(I M:_{R} M\right)=I$.

Proposition 1.8. Let $M$ be a graded finitely generated multiplication module over a G-graded commutative ring $R$. If $I$ is a graded $P$-primal ideal of $R$ containing $\left(0:_{R} M\right)$, then $I M$ is a graded $P$-primal submodule of $M$.

Proof. Clearly $\left.P=G(I)=G\left(I M:_{R} M\right)\right) \subseteq G(I M)$ by Lemma 1.3 and [5, Lemma 3.10]. Now assume that $a=\sum_{g \in G} a_{g} \in R$ is not $G$-prime to $I M$. Then, for every $g \in G$, there exists $m_{g^{\prime}} \in h(M) \backslash I M$ with $a_{g} m_{g^{\prime}} \in I M$. Then we have $R a_{g} R m_{g} \subseteq I M$. Since $M$ is graded multiplication, there exists a graded ideal $J$ of $R$ with $R m_{g^{\prime}}=J M$. Then from $\left(R a_{g} J\right) M=\left(R a_{g}\right)\left(R m_{g^{\prime}}\right) \subseteq I M$ we get $R a_{g} J \subseteq\left(I M:_{R} M\right)=I$ by Remark 1.7. If $J \subseteq I$, then $m_{g^{\prime}} \in R m_{g^{\prime}}=$ $J M \subseteq I M$ a contradiction. So there exists a homogeneous element $b_{\gamma} \in J \backslash I$. In this case $a_{g} b_{\gamma} \in R a_{g} J \subseteq I$ implies that $a_{g}$ is not homogeneous prime to $I$. 
Therefore $a$ is not $G$-prime to $I$, that is, $a \in P$. Hence $G(I M) \subseteq P$. Therefore $P=G(I M)$ and so $I M$ is graded $P$-primal.

Definition. Let $R$ be a $G$-graded commutative ring, and $M$ a graded $R$ module.

(1) We say that $R$ is a graded $P$-ring (or simply $g r$ - $P$-ring) if every proper graded ideal of $R$ is a finite product of graded primal ideals of $R$.

(2) $M$ is called a graded $P$-module (or simply $g r$ - $P$-module) if every graded proper submodule $N$ of $M$ has a graded primal factorization $N=I_{1} I_{2} \cdots I_{k} N^{*}$ where $I_{1}, I_{2}, \ldots, I_{k}$ are graded primal ideals of $R$ and $N^{*}$ is a graded primal submodule of $M$.

Theorem 1.9. Let $R$ be a $G$-graded commutative ring and $M$ a faithful graded finitely generated multiplication $R$-module. If $R$ is a gr-P-ring, then $M$ is a gr-P-module.

Proof. Assume that $R$ is a $g r$ - $P$-ring and $N$ is a proper graded submodule of $M$. Then $N=I M$ for some graded ideal $I$ of $R$. As $R$ is a $g r-P$-ring, $I$ has a factorization $I=I_{1} I_{2} \cdots I_{k}$ where $I_{i}$ is a graded $P_{i}$-primal ideal of $R$ $(1 \leq i \leq k)$. In this case $N=I M=\left(I_{1} I_{2} \cdots I_{k-1}\right)\left(I_{k} M\right)$. By Proposition 1.8, $I_{k} M$ is a graded primal submodule of $M$. So the result follows.

\section{Graded primal submodules of $M_{S}$}

Throughout this section $G$ will be a group. Let $S$ be a multiplicatively closed subset of homogeneous elements of $R$ and denote by $R_{S}$ the ring of fractions $S^{-1} R$. For $a / s \in R_{S}, a$ homogeneous, we set $\operatorname{deg}(a / s)=\operatorname{deg}(a)(\operatorname{deg}(s))^{-1}$. We further define a grading on $R_{S}$ by setting

$$
\left(R_{S}\right)_{i}=\left\{a / s \in R_{S}: a \text { is homogeneous and } \operatorname{deg}(a / s)=i\right\} .
$$

It is easy to see that $R_{S}$ is a graded ring. Also, for every graded ideal $I$ of $R$, $I_{S}=S^{-1} I$ is a graded ideal of $R_{S}$.

Now let $M=\sum_{g \in G} M_{g}$ be a graded $R$-module and consider the module of fractions $S^{-1} M$ and denote it by $M_{S}$. For every $m / s \in M_{S}$, with $m$ homogeneous, define $\operatorname{deg}(m / s)=\operatorname{deg}(m)(\operatorname{deg}(s))^{-1}$. Define a natural grading on $M_{S}$ by setting

$$
\left(M_{S}\right)_{i}=\left\{m / s \in M_{S}: m \text { is homogeneous and } \operatorname{deg}(m / s)=i\right\} .
$$

Then $M_{S}$ is a graded $R_{S}$-module.

The aim of this section is the studying of the relations between the graded primal submodules of $M$ and the graded primal submodules of $M_{S}$.

Lemma 2.1. Let $R$ be a $G$-graded commutative ring and $M$ a graded $R$-module. Let $S$ be a multiplicatively closed subset of homogeneous elements of $R$ and let $N$ be a graded $P$-primal submodule of $M$ with $P \cap S=\emptyset$. If $m / s \in N_{S}$, then $m \in N$. 
Proof. Suppose that $m / s \in N_{S}$. Without loss of generality we may assume that $m / s \in h\left(M_{S}\right)$. Then, there exist $n \in h(N)$ and $t \in S$ such that $m / s=n / t$. So $u t m=u s n \in N$ for some $u \in S$. If $m \notin N$, then $u t m \in N$ implies that $u t$ is not homogeneous prime to $N$, that is, $u t \in g(N) \cap S \subseteq P \cap S$ which is a contradiction.

Proposition 2.2. Let $R$ be a $G$-graded commutative ring and $M$ a graded $R$ module. Let $S$ be a multiplicatively closed subset of homogeneous elements of $R$ and let $N$ be a graded $P$-primal submodule of $M$ with $P \cap S=\emptyset$. If $K$ is a graded submodule of $M$, then $\left(N:_{R} K\right)_{S}=\left(N_{S}:_{R_{S}} K_{S}\right)$.

Proof. Clearly $\left(N:_{R} K\right)_{S} \subseteq\left(N_{S}: R_{S} K_{S}\right)$. For the reverse containment assume that $r / s \in\left(N_{S}: R_{S} K_{S}\right)$. Then, for every $m \in K$, we have $\mathrm{rm} / \mathrm{s}=$ $(r / s)(m / 1) \in N_{S}$. So, by Lemma 2.1, $r m \in N$. Therefore $r \in\left(N:_{R} K\right)$ and hence $r / s \in\left(N:_{R} K\right)_{S}$ and the result follows.

Let $R$ be a $G$-graded commutative ring, $M$ a graded $R$-module and $S$ a multiplicatively closed subset of homogeneous elements in $R$. Consider the canonical homomorphism $f: M \rightarrow M_{S}$ which is defined by $m \mapsto m / 1$. Then $f$ is a homogeneous homomorphism of degree $e$. If $\mathcal{N}$ is a graded submodule of $M_{S}$, define $\mathcal{N} \cap M=f^{-1}(\mathcal{N})$. Then we have the following proposition:

Proposition 2.3. Let $R$ be a G-graded commutative ring and $M$ a graded $R$-module. Let $N$ be a graded P-primal submodule of $M$, and let $S$ be a multiplicatively closed subset of homogeneous elements of $R$ with $P \cap S=\emptyset$. Then $N_{S}$ is a graded primal ideal of $M_{S}$ with adjoint ideal $P_{S}$. Furthermore $N=N_{S} \cap M$.

Proof. Clearly $P_{S}$ is a graded prime ideal of $R_{S}$. For every homogeneous element $a / s \in P_{S}$ we have $a \in P$. Hence $a$ is not homogeneous prime to $N$. Then, there exists $m \in h(M) \backslash N$ such that am $\in N$. As $m \in h(M) \backslash N$ we get $m / 1 \in h\left(M_{S}\right) \backslash N_{S}$ by Lemma 2.1. Hence $(a / s)(m / 1)=(a m) / s \in N_{S}$ implies that $a / s \in g\left(N_{S}\right)$. This implies that $P_{S} \subseteq G\left(N_{S}\right)$. Conversely, assume that $r / s \in g\left(N_{S}\right)$. Then, there exists $n / t \in h\left(M_{S}\right) \backslash N_{S}$ with $(r / s)(n / t) \in N_{S}$. Therefore $r n \in N$ with $n \in h(M) \backslash N$ by Lemma 2.1. This shows that $r \in$ $g(N) \subseteq G(N)=P$. Thus $r / s \in P_{S}$, that is, $G\left(N_{S}\right) \subseteq P_{S}$. We have already shown that $P_{S}$ consists exactly of elements of $R_{S}$ that are not $G$-prime to $N_{S}$. So $N_{S}$ is a graded $P_{S}$-primal submodule of $M_{S}$.

For the last part, it is obvious that $N \subseteq N_{S} \cap M$. To show that $N=N_{S} \cap M$, it suffices to show that $N_{S} \cap M \subseteq N$. Let $m \in N_{S} \cap M$. Then, from $m / 1 \in N_{S}$ we get $m \in N$ by Lemma 2.1 .

Proposition 2.4. Let $R$ be a $G$-graded commutative ring and $M$ a graded $R$ module. Let $S$ be a multiplicatively closed subset of homogeneous elements of $R$ and let $\mathcal{N}$ be a graded q-primal submodule of $M_{S}$, where $q \subseteq R_{S}$ is a graded prime ideal. Then $\mathcal{N} \cap M$ is a graded $(q \cap E)$-primal submodule of $M$. Moreover $(\mathcal{N} \cap M)_{S}=\mathcal{N}$. 
Proof. We know that $q \cap R$ is a graded prime ideal of $R$. It is enough to show that $G(\mathcal{N} \cap M)=q \cap R$. For every homogeneous element $a \in q \cap R$, $a / 1 \in q=G(\mathcal{N})$. So, there is $m / t \in h\left(M_{S}\right) \backslash \mathcal{N}$ with $(a / 1)(m / t) \in \mathcal{N}$. Hence $($ am $) / 1=($ tam $) / t \in \mathcal{N}$ implies that $a m \in \mathcal{N} \cap M$ with $m \in h(M) \backslash(\mathcal{N} \cap M)$. Hence $a \in g(\mathcal{N} \cap M) \subseteq G(\mathcal{N} \cap M)$. This implies that $q \cap R \subseteq G(\mathcal{N} \cap M)$. For the other containment, let $r \in g(\mathcal{N} \cap M)$. In this case there exists $m \in$ $h(M) \backslash(\mathcal{N} \cap M)$ with $r m \in \mathcal{N} \cap M$. Hence $m / 1 \in h\left(M_{S}\right) \backslash \mathcal{N}$ with $(r / 1)(m / 1)=$ $(\mathrm{rm}) / 1 \in \mathcal{N}$. This implies that $r / 1 \in g(\mathcal{N})$ and so $r / 1 \in G(\mathcal{N})=q$. Therefore $r \in q \cap R$. This implies that $G(\mathcal{N} \cap M) \subseteq q \cap R$. Therefore $G(\mathcal{N} \cap M)=q \cap R$.

Now we show that $(\mathcal{N} \cap M)_{S}=\mathcal{N}$. Clearly $\mathcal{N} \subseteq(\mathcal{N} \cap M)_{S}$. On the other hand, for every $m / s \in(\mathcal{N} \cap M)_{S}$ we have $m \in \mathcal{N} \cap M$ by Lemma 2.1 and so $m / 1 \in \mathcal{N}$. Let $m=\sum_{g \in G} m_{g}$. As $\mathcal{N}$ is graded we must have $m_{g} / 1 \in \mathcal{N}$ for every $g \in G$. Now from $\left(m_{g} / s\right)(s / 1)=m_{g} / 1 \in \mathcal{N}$ with $s / 1 \notin q$ we get $m_{g} / s \in \mathcal{N}$. Therefore $m / s \in \mathcal{N}$ shows that $(\mathcal{N} \cap M)_{S} \subseteq \mathcal{N}$. Thus $\mathcal{N}=(\mathcal{N} \cap M)_{S}$.

Theorem 2.5. Let $R$ be a G-graded commutative ring and $M$ a graded $R$ module. Let $P$ be a graded prime ideal of $R$ and $S$ a multiplicatively closed subset of homogeneous elements of $R$ with $P \cap S=\emptyset$. Then there exists a one-to-one correspondence between the graded P-primal submodules of $M$ and the graded $P_{S}$-primal submodules of $M_{S}$.

Proof. This follows from Propositions 2.3 and 2.4.

\section{The method of idealization}

Let $R$ be a commutative ring, and let $M$ be a unitary $R$-module. Then $R(+) M=R \oplus M$ (direct sum) with coordinate-wise addition and multiplication given by $\left(r_{1}, m_{1}\right)\left(r_{2}, m_{2}\right)=\left(r_{1} r_{2}, r_{1} m_{2}+r_{2} m_{1}\right)$ is a commutative ring with 1 (even an $R$-algebra) called the idealization of $M$ or the trivial extension of $R$ by $M$. Note that $R$ naturally embeds into $R(+) M$ by $r \rightarrow(r, 0)$, if $N$ is a submodule of $M$, then $0(+) N$ is an ideal of $R(+) M, 0(+) M$ is a nilpotent ideal of $R(+) M$ of index 2 , and that $(R(+) M) /(0(+) M) \approx R$. Moreover an ideal $J$ of $R(M)$ is a prime ideal if and only if $J=P \oplus M$ for some prime ideal $P$ of $R$. For any undefined terms here the reader may consult [9, Sec, 25] and $[1]$.

Let $R=\oplus_{g \in G} R_{g}$ be a $G$-graded commutative ring and $M=\oplus_{g \in G} M_{g}$ a graded $R$-module. Then $R(+) M=\oplus_{g \in G}\left(R_{g} \oplus M_{g}\right)$ and $\left(R_{g} \oplus M_{g}\right)\left(R_{g^{\prime}} \oplus M_{g^{\prime}}\right)=$ $R_{g} R_{g^{\prime}} \oplus\left(R_{g} M_{g^{\prime}}+R_{g^{\prime}} M_{g}\right) \subseteq R_{g g^{\prime}} \oplus M_{g g^{\prime}}$. Hence $R(+) M$ is a $G$-graded ring with $(R(+) M)_{g}=R_{g} \oplus M_{g}$. Consequently, $h(R(+) M)=\{(r, m) \mid r \in h(R), m \in$ $h(M)\}$.

Proposition 3.1. Let $R$ be a G-graded commutative ring and $M$ a G-graded $R$-module. The ideal $J$ of $R(+) M$ is graded prime if and only if $J=P(+) M$ where $P$ is a graded prime ideal of $R$. 
Proof. Assume first that $P$ is a graded prime ideal of $R$. Let $(a, m),\left(b, m^{\prime}\right) \in$ $h(R(+) M)$ be such that $(a, m)\left(b, m^{\prime}\right) \in P(+) M$. Then $a, b \in h(R)$ with $a b \in P$ gives $a \in P$ or $b \in P$. This implies that either $(a, m) \in P(+) M$ or $\left(b, m^{\prime}\right) \in$ $P(+) M$. So $P(+) M$ is a graded prime ideal of $R(+) M$. Conversely, assume that $J$ is a graded prime ideal of $R(+) M$. Then $J=P(+) N$ for some graded ideal $P$ of $R$ and some graded submodule $N$ of $M$ with $P M \subseteq N$. Suppose that $x, y \in h(R)$ are such that $x y \in P$. In this case $(x, 0),(y, 0) \in h(R(+) M)$ and $(x, 0)(y, 0)=(x y, 0) \in P(+) M$. It follows that either $(x, 0) \in P(+) M$ or $(y, 0) \in P(+) M$. So either $x \in P$ or $y \in P$, that is, $P$ is a graded prime ideal of $R$. Now assume that $N \neq M$ and choose $m \in h(M) \backslash N$ and $a \in P \cap h(R)$. Then $(a, m) \in h(R(+) M)$ and $a m \in P M \subseteq N$. Now $(a, m)^{2}=\left(a^{2}, a m+a m\right) \in$ $P(+) N=J$ but $(a, m) \notin P(+) N=J$ which contradicts the hypothesis that $J$ is a graded prime ideal. Consequently, $N=M$ and so $J=P(+) M$, as required.

Theorem 3.2. Let $R$ be a $G$-graded commutative ring, $M$ a $G$-graded $R$ module and $I$ a graded ideal of $R$. Then $I$ is a graded primal ideal of $R$ if and only if $I(+) M$ is a graded primal ideal of $R(+) M$.

Proof. For every $(a, m) \in g(I(+) M)$, there exists $\left(b, m^{\prime}\right) \in h(R(+) M) \backslash(I(+) M)$ such that $(a, m)\left(b, m^{\prime}\right) \in I(+) M$. In this case $a b \in I$ with $b \in h(R) \backslash I$, gives $a \in$ $g(I)$. Since every element of $G(I(+) M)$ is a sum of homogeneous elements of the form $(a, m), a \in h(R), m \in h(M)$, it follows that $G(I(+) M) \subseteq G(I)(+) M$. Conversely, assume that $(a, m)$ is a homogeneous element of $R(+) M$ with $a \in$ $g(I)$. Then $a b \in I$ for some $b \in h(R) \backslash I$. Now $(a, m)(b, 0)=(a b, b m) \in I(+) M$ with $(b, 0) \in h(R(+) M) \backslash I(+) M$ shows that $(a, m) \in g(I(+) M)$. As a consequence, $G(I)(+) M \subseteq G(I(+) M)$. Consequently, $G(I(+) M)=G(I)(+) M$. Now the result follows easily.

Theorem 3.3. Let $R$ be a G-graded commutative ring. Then the set of graded zero-divisors of $R$ is a union of graded prime ideals of $R$.

Proof. Let $\Sigma$ be the set of all graded ideals of $R$ in which every element is a graded zero-divisor, and partially order $\Sigma$ be the set theoretic inclusion. Then, by Zorn's Lemma, $\Sigma$ has a maximal member $P$ say. We show that $P$ is a graded prime ideal of $R$. Clearly $P \neq R$. If $P$ is not grade prime, there are $a, b \in h(R) \backslash P$ with $a b \in P$. By maximality of $P$, there exists an element $c \in P+R a$ which is not a graded zero-divisor. In this case $b c \in b P+R a b \subseteq P$. We claim that $P+R b \subseteq G z(R)$. Assume that $x=\sum_{g \in G} x_{g} \in P+R b$. If $x=0$, then $x \in G z(R)$. So assume that $x \neq 0$. Since $c$ is not a graded zero-divisor, there exists $h \in G$ such that $c_{h} w \neq 0$ for every nonzero $w \in h(R)$. Then we have $c_{h} x \in c_{h} P+R b c_{h} \subseteq P$ with $c_{h} x \neq 0$. As $P$ consists of graded zero-divisors, for every $g \in G$, there exists a nonzero $y_{g^{\prime}} \in h(R)$ with $c_{h} x_{g} y_{g^{\prime}}=0$. It follows that $x_{g} y_{g^{\prime}}=0$. This implies that $x \in G z(R)$. Therefore all elements of $P+R b$ are graded zero-divisors which contradicts the maximality of $P$. Therefore $P$ must be a graded prime ideal of $R$. 
Now let $r=\sum_{g \in G} r_{g} \in R$ be a graded zero-divisor. Let $\Omega$ be the subset of $\Sigma$ consisting of those graded ideals of $R$ which contain $r$. Then a similar argument as above shows that $\Omega$ has a maximal member which is a graded prime ideal of $R$. Thus $G z(R)$ is the union of all graded prime ideals of $R$.

Let $R$ be a $G$-graded commutative ring. The set of all $g r$-prime ideals of $R$ is called the graded (prime) spectrum of $R$ and is denoted by $\operatorname{Spec}^{g}(R)$. We write $\operatorname{rad}^{g}(R)$ for $\cap\left\{P \mid P \in \operatorname{Spec}^{g}(R)\right\}$, and call it the gr-prime radical of $R$. An element $a=\sum g \in G$ is called graded nilpotent if, for every $g \in G$, there exists a positive integer $n_{g}$ with $a_{g}^{n_{g}}=0$ [11]. Then $G r(0)$ is the set of all graded nilpotent elements of $R$. It is well known that $\operatorname{rad}^{g}(R)=G r(0)$. As a consequence we get $G r(0) \subseteq G z(R)$.

Theorem 3.4. Let $R$ be a G-graded commutative ring and $M$ a graded $R$ module. Then

(1) $\{(r, m) \mid r \in G z(R) \cup G z(M), m \in M\} \subseteq G z(R(+) M)$.

(2) If $G z(R) \subseteq G z(M)$ or $G z(M) \subseteq G z(R)$, then $G z(R(+) M)=\{(r, m) \mid$ $r \in G z(R) \cup G z(M), m \in M\}$.

Proof. (1) Assume that $r=\sum_{g \in G} r_{g} \in G z(R) \cup G z(M)$. If $r \in G z(R)$, then, for every $g \in G$, there exists a nonzero $s_{g^{\prime}} \in h(R)$ with $r_{g} s_{g^{\prime}}=0$. In this case, $\left(s_{g^{\prime}}, 0\right) \in h(R(+) M)$ and $\left(r_{g}, 0\right)\left(s_{g^{\prime}}, 0\right)=(0,0)$. This implies that $(r, 0) \in$ $G z(R(+) M)$. If $r \in G z(M)$, then, for every $g \in G$, there exists $m_{g^{\prime}} \in h(M)$ with $r_{g} m_{g^{\prime}}=0$. Then from $\left(0, m_{g^{\prime}}\right) \in h(R(+) M)$ and $\left(r_{g}, 0\right)\left(0, m_{g^{\prime}}\right)=(0,0)$ we get $(r, 0) \in G z(R(+) M)$. Now assume that $m=\sum_{g \in G} m_{g} \in M$ is an arbitrary element. As, for every $g \in G,\left(0, m_{g}\right)^{2}=(0,0)$ we get $(0, m) \in$ $\operatorname{rad}^{g}(R(+) M)$. Now we have $(r, m)=(r, 0)+(0, m) \in G z(R(+) M)$ (Note that this follows since $G z(R(+) M)$ is a union of graded prime ideals and $\operatorname{rad}^{g}(R(+) M)$ is contained in each graded prime ideals).

(2) Assume that $(r, m)=\sum_{g \in G}\left(r_{g}, m_{g}\right) \in G z(R(+) M)$. Then, for every $g \in$ $G$, there exists a nonzero element $\left(s_{g}, n_{g}\right) \in h(R(+) M)$ with $\left(r_{g}, m_{g}\right)\left(s_{g}, n_{g}\right)=$ $(0,0)$. If $s_{g} \neq 0$, then $r_{g} \in G z(R)$ and if $s_{g}=0$, then $r_{g} \in G z(M)$. If $G z(R) \subseteq G z(M)$, then $r_{g} \in G z(M)$ for every $g \in G$. Thus $(r, m) \in\{(r, m) \mid r \in$ $G z(R) \cup G z(M)\}$ and if $G z(M) \subseteq G z(R)$, then $r_{g} \in G z(R)$ for every $g \in G$, that is, $(r, m) \in\{(r, m) \mid r \in G z(R) \cup G z(M)\}$. Therefore $G z(R(+) M \subseteq\{(r, m) \mid r \in$ $G z(R) \cup G z(M)\}$. So we have the equality.

Definition. Let $R$ be a commutative graded ring. We say that $R$ has a few graded zero-divisors if the set $G z(R)$ of graded zero-divisors of $R$ is a finite union of graded prime ideals.

Theorem 3.5. Let $R$ be a G-graded commutative ring and $M$ a graded $R$ module such that $R(+) M$ has a few graded zero-divisors and either $G z(R) \subseteq$ $G z(M)$ or $G z(M) \subseteq G z(R)$. Let $I$ be a graded ideal of $R$ and $N$ a graded submodule of $M$. Then $I(+) N$ is a graded primal ideal of $R(+) M$ if and only if either 
(a) $N=M$ and $I$ is a graded primal ideal of $R$ or

(b) $N \neq M, I M \subseteq N$, and $I$ and $N$ are graded $P$-primal where $P=G(I)$. In either case, $I(+) N$ is graded $P(+) M$-primal.

Proof. Assume that $N=M$. Then, by Theorem 3.2, $I(+) M$ is a graded primal ideal of $R(+) M$ with the adjoint ideal $P(+) M$ if and only if $I$ is a graded primal ideal of $R$ with the adjoint ideal $P$. So assume that $N \neq M$. For $I(+) N$ to be an ideal of $R(+) M$, we must have $I M \subseteq N$. By passing to $(R(+) M) /(I(+) M)$, we can assume that $I=0$ and $N=0$. So what we required is to show that $R(+) M$ is a graded primal ring if and only if both $R$ is a graded $P$-primal ring and $M$ is a graded $P$-primal $R$-module where $P=G(0)$. Now, by Theorem 1.5, $R(+) M$ is a graded primal ring if and only if $G z(R(+) M)$ is a graded prime ideal of $R(+) M$, or equivalently, by Theorem 3.4, $(G z(R) \cup G z(M))(+) M$ is a graded prime ideal of $R(+) M$, if and only if $G z(R) \cup G z(M)$ is a graded prime ideal of $R$ by Proposition 3.1. Since $G z(R)$ and $G z(M)$ are each a union of graded prime ideals of $R, R(+) M$ has a few graded zero-divisors, and since $G z(R) \cup G z(M)$ is a graded prime ideal, $G z(R) \cup G z(M)$ is a graded ideal of $R$ if and only if $G z(R)=G z(M)=P$ for some graded prime ideal $P$ of $R$, that is, both $R$ and $M$ are graded $P$-primal.

Acknowledgments. I am grateful to the referees for taking the time to carefully read the paper, and for making many perspicacious comments which improved the earlier version of the paper a lot.

\section{References}

[1] D. D. Anderson and M. Winders, Idealization of a module, J. Commut. Algebra 1 (2009), no. $1,3-56$.

[2] J. Dauns, Primal modules, Comm. Algebra, 25 (1997), no. 8, 2409-2435.

[3] S. Ebrahimi Atani, On graded weakly prime ideals, Turkish J. Math. 30 (2006), no. 4, $351-358$.

[4] _ On graded prime submodules, Chaing Mai J. Sci. 33 (2006), no. 1, 3-7.

[5] S. Ebrahimi Atani and R. Ebrahimi Atani, Graded multiplication modules and the graded ideal $\theta_{g}(M)$, Turk. J. Math. 33 (2009), 1-9.

[6] S. Ebrahimi Atani and F. Farzalipour, On graded multiplication modules, Chiang Mai J. Sci., To appear.

[7] S. Ebrahimi Atani and A. Yousefian Darani, Graded primal ideals, Georgian Math. J., Ahead of print.

[8] L. Fuchs, On primal ideals, Proc. Amer. Math. Soc. 1 (1950), 1-6.

[9] J. A. Huckaba, Commutative Rings with Zero-Divisors, monographs and textbooks in Pure and Applied mathematics, 117, Marcel Dekker, Inc., Now York, 1988.

[10] C. Nastasescu and F. Oystaeyen, Methods of Graded Rings, Lecture Notes in Mathematics, 1836. Springer-Verlag, Berlin, 2004.

[11] M. Refai and K. Al-Zoubi, On Graded Primary Ideals, Turkish J. Math. 28 (2004), no. 3, 217-229.

[12] R. Y. Sharp, Asymptotic behaviour of certain sets of attached prime ideals, J. London Math. Soc. (2) 34 (1986), no. 2, 212-218.

[13] P. Smith, Some remarks on multiplication modules, Arch. Math. 50 (1988), no. 3, 223235 . 
Department of Mathematics

University of Mohaghegh Ardabili

P.O. Box 179, Ardabil, Iran

E-mail address: yousefian@uma.ac.ir, youseffian@gmail.com 